\title{
Evaluation with endothelial nitric oxide synthase (eNOS) immunoreactivity of the protective role of astaxanthin on hepatorenal injury of remote organs caused by ischaemia reperfusion of the lower extremities
}

\author{
Ahmet Uyar ${ }^{1}$, Turan Yaman² \\ ${ }^{1}$ Department of Pathology, Hatay Mustafa Kemal University, Veterinary Faculty, Hatay, Turkey \\ 2Department of Pathology, Van Yuzuncu Yil University, Veterinary Faculty, Van, Turkey
}

Gastroenterology Rev 2020; 15 (2): 161-172

DOI: https://doi.org/10.5114/pg.2019.88620

Key words: hepatorenal ischaemia reperfusion injury, astaxanthin, endothelial nitric oxide synthase.

Address for correspondence: Assist. Prof. Dr. Ahmet Uyar, Department of Pathology, Veterinary Faculty, Mustafa Kemal University, 31040, Hatay, Turkey, phone: +90 3262455313, fax: +903262455704, e-mail: uyarahmet@hotmail.com

\begin{abstract}
Introduction: Ischemia and following reperfusion triggers local and systemic damage with the involvement of free oxygen radicals and inflammatory mediators. Although blood flow saves extremity from necrosis, multi organ dysfunction may progress and cause death of the patient.

Aim: The study aims to examine the effect of astaxanthin (AST) on the prevention of remote tissue injury resulting from lower extremity ischaemia-reperfusion (I/R). To elucidate the potential hepatoprotective and renoprotective effects of AST, in addition to histopathological findings, the intrahepatic and intrarenal kinetics of endothelial nitric oxide synthase (eNOS) during I/R were determined by using the immunohistochemical method.

Material and methods: Twenty-eight male Wistar albino rats were divided into four groups. For the control group, only the anaesthesia procedure ( $2 \mathrm{~h}$ ) was conducted without I/R. In the I/R group, $2 \mathrm{~h}$ of reperfusion was conducted following ischaemia under anaesthesia. For the I/R group + AST, 7 days prior to ischaemia, $125 \mathrm{mg} / \mathrm{kg}$ AST was given with gavage, and $2 \mathrm{~h}$ of ischaemia and $2 \mathrm{~h}$ of reperfusion were conducted under anaesthesia. Following necropsy, liver and kidney tissue samples were fixed in $10 \%$ buffered formalin for $48 \mathrm{~h}$ for histopathological and immunohistochemical investigation.

Results: The histological analysis revealed that severe I/R hepatorenal injury such as inflammatory cell infiltration, dilatation in sinusoids and lumen of tubuli, congestion in glomerular capillaries, degeneration in hepatocyte and epithelial cells of tubuli, and necrosis was ameliorated by AST. Immunohistochemical studies showed that the I/R-induced elevation in eNOS expression was reduced by AST treatment.

Conclusions: In the case of acute lower extremity I/R, AST decreased the ischaemic injury in liver and renal tissues by protecting the microcirculation and providing a cytoprotective effect with vasodilatation.
\end{abstract}

\section{Introduction}

Ischaemia and the subsequent reperfusion decrease the effectiveness and success of such treatments as by-pass surgery and transplantations of microvascular tissues, organs, and extremities as well as in cases of shock, burns, sepsis, pancreatitis, cerebrovascular events, myocardial infarct, and orthopaedic and cardiovascular surgical interventions [1-3]. Ischaemia is defined as the interruption of the maintenance of oxygen and other metabolites by circulation and the insufficiency of the removal of metabolic end products [4]. Reperfusion is the reversal of this interruption of blood circulation [5]. The main reasons for the damage occurring in ischaemia-reperfusion (I/R) are oxidative damage due to oxidative burst by the recovered circulation and oxidative damage caused by polymorphonuclear leucocytes during the inflammatory response 
[6]. Following acute extremity I/R, massive local tissue damage and systemic complications arise. The death rate and the amputation rate due to this condition were reported as $25-50 \%$ and $15-40 \%$, respectively [7].

The intensity of tissue damage caused by ischaemia is affected by various factors, such as width of clogged artery, clogging period, and characteristic properties of tissue $[2,8]$. According to the period of ischaemia, symptomatic subclinical damage, oedema, degeneration, necrosis, distant organ function loss, and multi-organ failure symptoms may occur in I/R in the target and end organs, such as lung, kidney, liver, heart, brain, and intestines. These conditions may lead to mortality [3, 9, 10]. One of the important consequences of I/R damage is end-organ damage, which results in high mortality and morbidity. Lung, liver, and kidney failures and dysfunctions of the central nervous system, gastrointestinal system, and myocardia initially occur [2]. Therefore, damage occurring in ischaemic tissue after reperfusion has been investigated in various organs, such as lung, kidney, heart, brain, and intestines [3, 9, 10].

Nitric oxide (NO) is synthesised from amino acid L-arginine through nitric oxide synthase (NOS) [11] and has the following isoforms: neuronal nitric oxide synthase (nNOS), inducible nitric oxide synthase (iNOS), and endothelial nitric oxide synthase (eNOS) [12]. NO, which is produced in the endothelial vessel, relaxes the vessel smooth muscle and plays a role in various important events, such as blood flow, regulation of blood pressure, prevention of thrombocyte adhesion and aggregation, suppression of vessel smooth muscle proliferation and migration, contraction of the heart muscle, and regulation of the gastrointestinal system [13-15].

Following the discovery of $\mathrm{NO}$ in mammalian cells as an endogenous molecule, its physiological roles in the cardiovascular, respiratory, urinary, gastrointestinal, and nervous systems have been examined [16]. The expression of eNOS increases because of the shear stress caused by increased arterial blood flow. Accordingly, $\mathrm{NO}$ is released to provide vascular relaxation. When NO cannot be released in a certain amount, endothelial dysfunction occurs, which leads to hypertension and other cardiovascular diseases $[11,15]$. The level of NO changes in many diseases, such as chronic viral hepatitis, autoimmune liver disease, hepatocarcinoma, kidney malfunction, hypertension, diabetes, atherosclerosis, pulmonary hypertension, venereal tumours, pregnancy, and septic shock $[11,13,14]$. The main goal of reperfusion is to protect tissues or organs and repair them to regain their functions [2, 17]. Treatments fail and costs increase when the prevention of ischaemia and the subsequent reperfusion damage is not possible in clinical procedures; this situation may end up with the loss of the patient.
Therefore, intensive scientific research efforts must be conducted on the prevention of I/R damage.

In histopathological examination of I/R polymorphonuclear cell infiltration, hepatocellular necrosis, and sinusoidal enlargement, sinusoidal congestion, cytoplasmic vacuolisation and haemorrhage were seen [18-21]. Liver I/R damage is caused by different mechanisms effecting liver damage at different levels. Kupffer cell activation, formation of reactive oxygen species (ROS), release of cytokines and chemokines, vasoconstriction, deterioration of the balance between $\mathrm{NO}$ and endothelin balance, accumulation of neutrophil leucocytes, alteration of mitochondrial permeability, and $\mathrm{pH}$ paradox are among the listed cellular and molecular interactions. These complex mechanisms cause cell death, organ function loss, and organ loss. When liver I/R damage is investigated under a light microscope, neutrophil leucocyte (NL) infiltration, regional haemorrhage and necrosis, congestion, enlargement in sinusoids, focal hepatocellular vacuolisation, and hepatocyte swelling are observed. At the ultrastructural level, deterioration in the mitochondrial structure, swelling, alterations in staining, and accumulation of NL are observed [22-24].

The kidney is an organ with high perfusion and is therefore sensitive to hypoperfusion. Accordingly, I/R damage is commonly encountered in the kidneys and leads to serious consequences. The reason for acute kidney failure in aortic surgery is mostly I/R damage, which increases morbidity and mortality in the postoperative period [1, 2]. I/R damage primarily begins from the hypoxia-sensitive region of the kidney. Much of the renal flow entering into the kidney passes from the renal cortex and to the lower vasa recta, which perfuses the renal medulla and makes it more prone to hypoxia [3, 25]. In addition, medullar hypoxia reduces the cellular energy stores and deteriorates the actin cytoskeleton found in endothelial and smooth muscle cells. It causes cellular deformation and increases hypoxia in adjacent tissues [26]. Renal damage initially occurs in the tubules because of tubular necrosis due to ischaemia [27]. Acute kidney failure due to ischaemia is characterised by decreased glomerular filtration rate, blurred swelling in the tubuli epithelium, acute tubular necrosis, inflammatory cellular infiltration, oedema, congestion, and increased resistance in the kidney vessels [28]. The interruption of kidney blood flow or its attenuation and the subsequent reperfusion cause a certain degree of tissue damage [29].

Currently, many treatment procedures for preventing and ameliorating I/R damage are being developed. However, I/R may still cause mortality [30]. Scientists have recently used antioxidants other than substances 
such as immunosuppressives and corticoids. Antioxidants, which have gained popularity recently, scavenge the free radicals caused by $I / R$ and decrease $I / R$ damage [31, 32].

Astaxanthin (AST) is a carotenoid obtained from microalgae, and it has beneficial effects on human health because of its powerful antioxidant property. Currently, the most potent microbial source in AST production is Haematococcus microalgae. AST is widely used in the maintenance of colours of pet tropical fish, the colouring of poultry eggs, and food supplements because of its beneficial effect on the metabolism [33, 34].

\section{Aim}

In this study, we aimed to investigate the damage of lower extremity ischemia/reperfusion on remote organs, liver and kidney, and the preventative effect of AST, a potent antioxidant, by histopathological and immunohistochemical methods.

\section{Material and methods}

Twenty-eight male Wistar albino rats, weighing 250-300 g (7-8 weeks of age) were used in the experiments. The animals were kept at a constant temperature of $20-22^{\circ} \mathrm{C}$ and a 12 -hour light-dark cycle (lights on 7:00-19:00; darkness 19:00-7:00). All animals were kept in stainless cages and fed with a standard pellet diet. Food and water were given ad libitum. Following an adaptation period of 1 week, the trials were started. The animals received humane care according to the criteria outlined in the 'Guide for the Care and Use of Laboratory Animals' prepared by the National Academy of Science and published by the National Institutes of Health. The ethics regulations were followed in accordance with the National and Institutional Guidelines for the Protection of Animal Welfare During Experiments. All animal experiments were performed in Bingöl University Animal Experiment Facility. The practices on animals throughout this study were approved by the Ethics Committee of the Bingöl University (Protocol number: 2015/08).

The rats were divided into four groups of eight rats.

Group I $(n=7)$ - control group: only anaesthesia procedure $(2 \mathrm{~h})$ was conducted in this group without ischaemia-reperfusion.

Group II $(n=7)$ : ischaemia/reperfusion (I/R): $2 \mathrm{~h}$ of reperfusion was conducted following ischaemia $(2 \mathrm{~h})$ under anaesthesia.

Group III $(n=7)$ : ischaemia/reperfusion (I/R) + AST (125 mg/kg) group: 7 days prior to ischaemia $125 \mathrm{mg} / \mathrm{kg}$ AST were given with gavage. Two hours of ischaemia and $2 \mathrm{~h}$ of reperfusion were conducted under anaesthesia.

Group IV ( $n=7)$ : AST $125 \mathrm{mg} / \mathrm{kg}$ group: $125 \mathrm{mg} / \mathrm{kg}$ AST was given with gavage for 7 days. Then anaesthesia was given for $2 \mathrm{~h}$ without ischaemia.

The procedures mentioned were conducted under anaesthesia with $50 \mathrm{mg} / \mathrm{kg}$ ketamine hydrochloride + $10 \mathrm{mg} / \mathrm{kg}$ xylazine hydrochloride.

\section{Histopathological and}

\section{immunohistochemical investigations}

Necropsy of rats was performed at the end of the study, and tissue samples were fixed in $10 \%$ buffered formalin solution for $48 \mathrm{~h}$ for histopathological and immunohistochemical investigation. Under routine follow-up, tissue samples were passed through alcohol series and dehydrated. They were passed through xylol series and blocked in paraffin. From those blocks some of the cross sections cut in $4 \mu \mathrm{m}$ thickness with a microtome (Leica RM 2135) were stained with Haematoxilen-Eosin (H.E.) dye. Histopathologically the presence of inflammatory cell infiltration, haemorrhage, dilatation in sinusoids and lumen of tubuli, congestion in glomerular capillaries, degeneration in hepatocyte and tubular epithelial cells, and necrosis was investigated in liver and kidney. In addition, narrowing in Bowman capsule and findings such as adhesion were semi-quantitatively assessed in kidney.

For immunohistochemical assessment cross sections obtained in $4 \mu \mathrm{m}$ thickness were placed in poly-L-lysine slides. In order to show eNOS immune reactivity anti-eNOS primary antibody (Abcam, ab3523 $1 / 200$ diluation, UK) was used and stained with avidin-biotin peroxidase complex $(A B C)$ immunohistochemical method. The obtained cross sections were kept in a heater for $1 \mathrm{~h}$ at $60^{\circ} \mathrm{C}$ and made pellucid in two different xylol solutions. Then they were dehydrated in a decreasing alcohol series and finally kept in distilled water for $5 \mathrm{~min}$. Cross sections were kept in citrate buffer solution in an ambient temperature for $20 \mathrm{~min}$. In order to avoid endogenous peroxidase sections were kept in 3\% hydrogen peroxide solution for $5 \mathrm{~min}$. They were washed for 5 min three times with PBS and kept overnight at $4^{\circ} \mathrm{C}$ after dropping eNOS primary antibody. The following day, sections were washed three times with PBS then incubated with biotinylated secondary antibody for $30 \mathrm{~min}$. After dropping the secondary antibody (Thermo Fisher Scientific, USA), staining substance diaminobenzidine (DAB chromogen (Thermo Fisher Scientific, USA)) was applied on antibody-biotin-avidin-peroxidase complex to make them visible. Background staining was performed with Mayer's haematoxylin. Following dehydration in graded alcohols, 
sections were made pellucid and then covered with Entellan. The intensity of immunohistochemical staining was evaluated by pathologists. eNOS staining intensity was scored as (-) in no staining, (+) in low staining, $(++)$ in moderate staining, and (+++) in intense staining. All samples were investigated and photographed under a Nikon 80i-DS-RI2 research microscope.

\section{Results}

No significant damage in the liver and kidney macroscopy was observed in the experimental groups.

A normal histological appearance in the liver was observed in the control group and the AST-only group during the microscopic investigation. Hepatocytes and portal areas were normal in appearance; the hepatocytes were found in the regular remark cord around the vena centralis in these two groups. Sinusoids within the remark cord were normal in diameter and structure. No inflammatory cell infiltration was observed pathologically (Figures $1 \mathrm{~A}$ and $\mathrm{B}$ ). In the I/R group, disruption of the integrity of remark cord in the lobules and a focal parenchymatic and hydropic degeneration distributed randomly in the liver parenchyma. In addition, hepatocytes with pycnotic nucleus, which have eosinophilic-stained cytoplasm, karyorrhexis and karyolysis developed into coagulation necrosis in some of the lobules. Vasa-congestion and sinusoidal dilatation in the focal form were also observed. Mononuclear cell infiltration was seen in the areas of periportal and periacinary (Figure $1 \mathrm{C}$ ). In the I/R + AST group, only a few low-intensity congestions were observed in the vena centralis and portal veins. Generally, the hepatic cord structure in the lobules was intact and similar to that in the control group. In addition, a low level of dilatation in the sinusoids and an insignificant level of sinusoidal hyperaemia compared with that in the I/R group were observed in some of the lobules. Degeneration in the hepatocytes were seen rarely, but necrosis and mon-
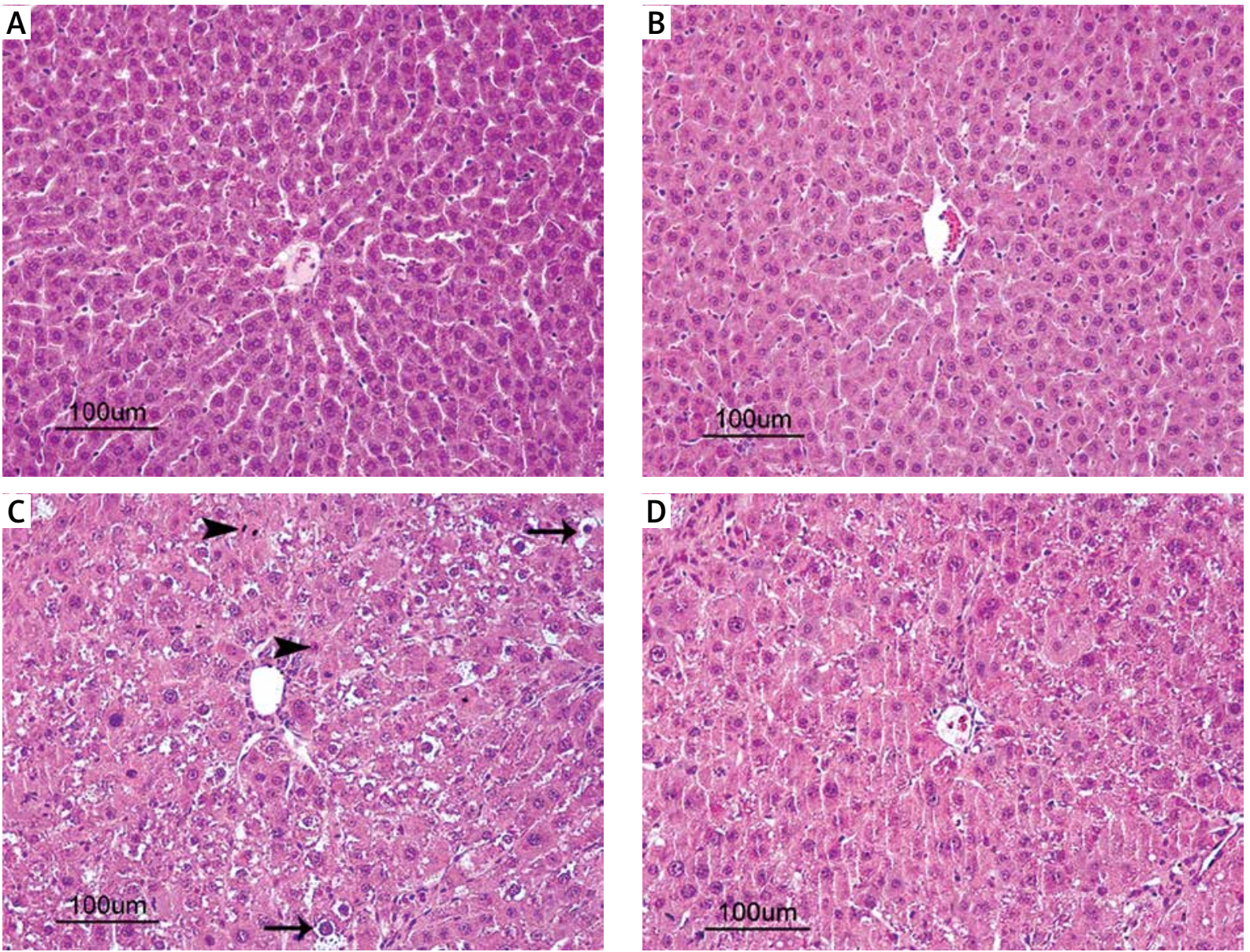

Figure 1. Photomicrographs of H\&E-stained histological slides of the liver. A, B - Normal histological appearance of liver in the control group and AST group. C - Appearance of parenchymatic and hydropic degeneration (arrow) and necrosis (head arrow) of severely affected liver in the I/R group. D - Appearance of degeneration and necrosis of slightly effected liver in the I/R + AST group 
Table I. Protective effect of astaxanthin on liver microscopic structure in experimental ischaemia reperfusion in rats

\begin{tabular}{|c|c|c|c|c|}
\hline Parameter (liver) & Control & I/R & $\mathrm{I} / \mathrm{R}+\mathrm{AST}$ & AST \\
\hline Periportal and periacinary inflammatory cell infiltration: & $-/ 7$ & $7 / 7$ & $3 / 7$ & -17 \\
\hline Low & - & 1 & 2 & - \\
\hline Moderate & - & 3 & 1 & - \\
\hline Intense & - & 3 & - & - \\
\hline Distruption in hepatic cordon structure: & $-/ 7$ & $7 / 7$ & $3 / 7$ & -17 \\
\hline Low & - & - & 1 & - \\
\hline Moderate & - & 1 & 2 & - \\
\hline Intense & - & 6 & - & - \\
\hline Dilatation and hyperaemia in sinusoids: & $-/ 7$ & $7 / 7$ & $3 / 7$ & $1 / 7$ \\
\hline Low & - & - & 2 & 1 \\
\hline Moderate & - & - & 1 & - \\
\hline Intense & - & 7 & - & - \\
\hline Parenchymal and hydropic degeneration: & -17 & $7 / 7$ & $3 / 7$ & -17 \\
\hline Low & - & - & 1 & - \\
\hline Moderate & - & 3 & 2 & - \\
\hline Intense & - & 4 & - & - \\
\hline Necrosis: & -17 & $7 / 7$ & $2 / 7$ & -17 \\
\hline Low & & 1 & 1 & - \\
\hline Moderate & - & 4 & 1 & - \\
\hline Intense & - & 2 & - & - \\
\hline Congestion in vena centralis and portal veins: & -17 & $7 / 7$ & $3 / 7$ & $-/ 7$ \\
\hline Low & - & 1 & 2 & - \\
\hline Moderate & - & 3 & 1 & - \\
\hline Intense & - & 3 & - & - \\
\hline
\end{tabular}

onuclear cell infiltration were not found (Figure $1 \mathrm{D})$. The results of the histopathological findings of the liver tissue are given in Table I.

No histopathological finding was observed when the kidneys of the control group and the AST-only group were compared histopathologically under a microscope. The tubular epithelial cells and glomerular structures were regular in appearance without any degeneration and necrosis (Figures $2 \mathrm{~A}$ and $\mathrm{B}$ ). Kidney tissues of the I/R group showed a significant swelling of the cytoplasm of the tubular epithelial cells, paling of colour due to hydropic degeneration, nuclei of some epithelial cells undergo necrosis and become picnotic and dilatation of tubular lumens were observed. Enlargement of the Bowman capsules of some glomeruli and a sclerotic structure caused by the narrowing of the Bowman capsules were apparent. Adhesion of the ba-sal membranes of some glomeruli, which caused the narrowing in the Bowman capsules, was observed. In addition, prominent hyperaemia in the glomerular capillaries and intertubular capillaries was observed. Focal inflammatory cells were found in the intertubular region (Figure $2 \mathrm{C}$ ). In the I/R + AST group, minimal hydropic degeneration in the tubular epithelial cells and necrosis were observed. In some of the glomeruli and intertubular capillaries, minimal level of hyperaemia was seen (Figure 2 D). Generally, the tubular and glomerular structures were close to normal histology. The results of the histopathological findings of the kidney tissue are given in Table II. 

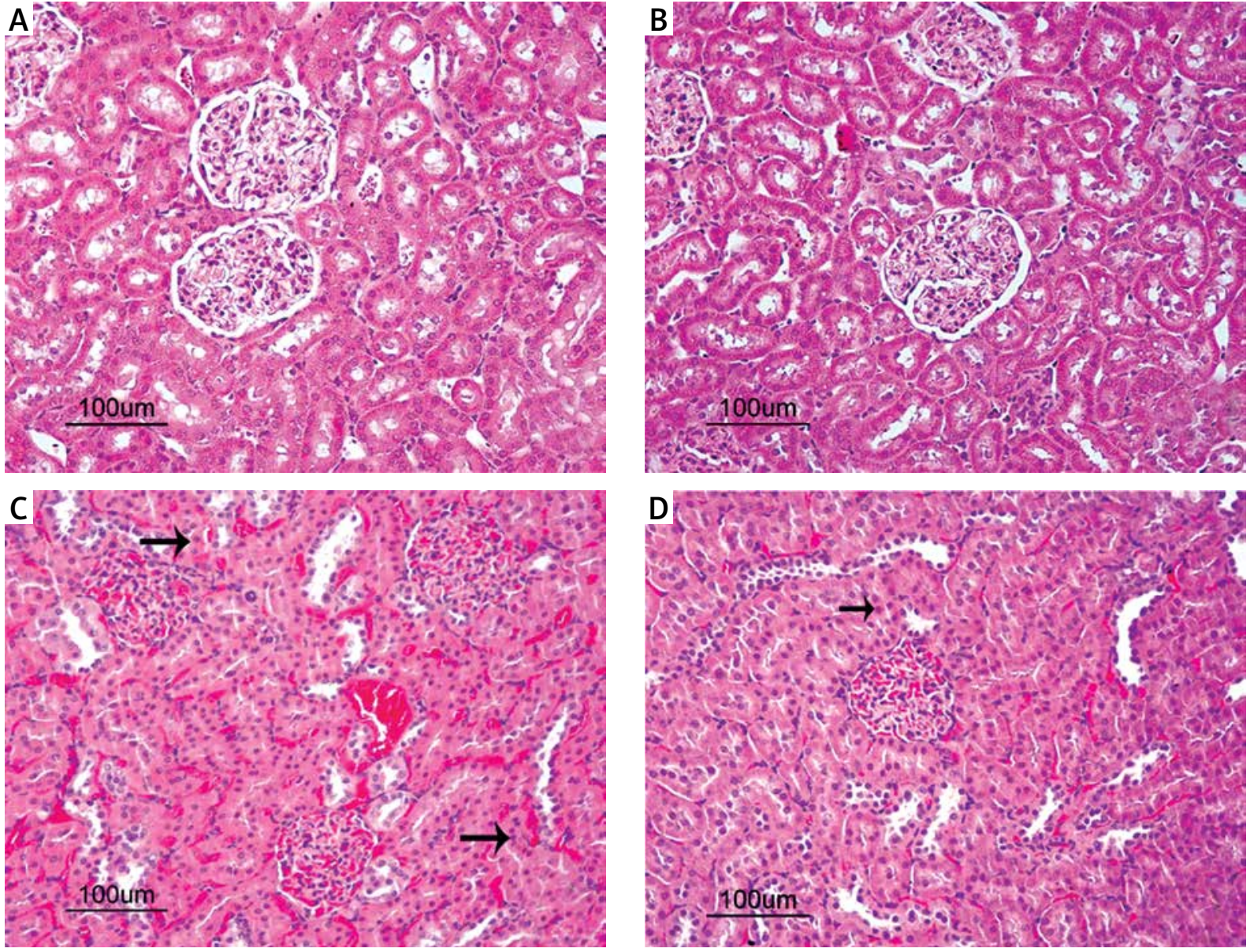

Figure 2. Photomicrographs of H\&E-stained histological slides of the kidney. A, B - Normal histological appearance of kidney in the control group and AST group. $\mathbf{C}$ - Appearance of adhesion of the basal membranes, degeneration and necrosis of tubuli (arrow), hyperaemia and congestion of severely affected kidney in the I/R group. D - Appearance of minimal degeneration and necrosis of slightly effected kidney in the $\mathrm{I} / \mathrm{R}+\mathrm{AST}$ group

In the immunohistochemical investigation of the eNOS activity in liver, no staining was seen in the control group (Figure $3 \mathrm{~A}$ ) and the AST-only group (Figure $3 \mathrm{~B}$ ). However, in the I/R group, a strong eNOS activity at the +++ level was observed in the sinusoidal capillaries (Figure $3 \mathrm{C})$, whereas a low eNOS reactivity at the + level was seen in the I/R + AST group (Figure $3 \mathrm{D}$ ).

In the investigation of the kidney eNOS reactivity using immunohistochemical methods, no staining was observed in the control group (Figure $4 \mathrm{~A}$ ) and the AST-only group (Figure $4 \mathrm{~B}$ ). In the I/R group (Figures $4 \mathrm{C}$ ), a potent eNOS activity at the +++ level was seen in both glomerular capillaries and intertubular capillaries. However, in the I/R + AST group, minimal eNOS activity at the + level was observed (Figure $4 \mathrm{D}$ ). The results of the immunohistochemical investigation and scores in terms of eNOS activity in liver and kidney tissue are given in Table III and Figure 5.

\section{Discussion}

If blood supply to the tissue is insufficient or stops, tissue perfusion is hampered and oxygen supply is diminished. Prolonging this condition causes a disorder in the functioning of the cells in this tissue and leads to the loss of cell integrity and even cell death [35].

This study aimed to investigate the effect of a potent antioxidant called AST on liver and kidney tissues in lower extremity I/R using histopathological and immunohistochemical methods. Because free radicals form quickly, just 15-20 s after reperfusion, any radical scavenger used for the prevention of reperfusion damage must be given at least $15 \mathrm{~min}$ prior to reperfusion. Thus, AST was administered for 7 days before I/R and given daily in a dose of $125 \mathrm{mg} / \mathrm{kg}$.

Many studies have used different agents to avoid reperfusion damage in liver. Lin et al. [36] revealed that 
Table II. Protective effect of astaxanthin on kidney microscopic structure in experimental ischaemia reperfusion in rats

\begin{tabular}{|c|c|c|c|c|}
\hline Parameter (kidney) & Control & I/R & $\mathrm{I} / \mathrm{R}+\mathrm{AST}$ & AST \\
\hline Glomerular sclerosis and adhesion: & $-/ 7$ & $7 / 7$ & $3 / 7$ & -17 \\
\hline Low & - & 1 & 2 & - \\
\hline Moderate & - & 3 & 1 & - \\
\hline Intense & - & 3 & - & - \\
\hline Dilatation of Bowman capsule: & $-/ 7$ & $7 / 7$ & $3 / 7$ & $-/ 7$ \\
\hline Low & - & - & 1 & - \\
\hline Moderate & - & 1 & 2 & - \\
\hline Intense & - & 6 & - & - \\
\hline Dilatation of tubules: & -17 & $7 / 7$ & $3 / 7$ & $1 / 7$ \\
\hline Low & - & - & 2 & 1 \\
\hline Moderate & - & - & 1 & - \\
\hline Intense & - & 7 & - & - \\
\hline Parenchymal and hydropic degeneration: & -17 & $7 / 7$ & $3 / 7$ & $-/ 7$ \\
\hline Low & - & - & 1 & - \\
\hline Moderate & - & 3 & 2 & - \\
\hline Intense & - & 4 & - & - \\
\hline Necrosis of tubular epithelium: & $-/ 7$ & $7 / 7$ & $2 / 7$ & $-/ 7$ \\
\hline Low & & 1 & 1 & - \\
\hline Moderate & - & 4 & 1 & - \\
\hline Intense & - & 2 & - & - \\
\hline Hyperaemia in glomerular and intertubular capillaries: & $-/ 7$ & $7 / 7$ & $3 / 7$ & $-/ 7$ \\
\hline Low & - & 1 & 2 & - \\
\hline Moderate & - & 3 & 1 & - \\
\hline Intense & - & 3 & - & - \\
\hline Intercellular inflammatory cell infiltration: & $-/ 7$ & $7 / 7$ & $3 / 7$ & $-/ 7$ \\
\hline Low & - & 1 & 2 & - \\
\hline Moderate & - & 3 & 1 & - \\
\hline Intense & - & 3 & - & - \\
\hline
\end{tabular}

curcumin administration exerted a moderate positive effect on hepatic damage. Ahmetova [37] observed hepatocytes with lost integrity, dilatation in the sinusoids, mononuclear cell infiltration, hydropic degeneration in the hepatocytes, and focal necrosis in some areas. Conversely, in the levosimendan group, regular integrity of the hepatocyte cords, less dilatation in the sinusoids, less mononuclear cell infiltration, and less hydropic degeneration and focal necrosis were observed in the hepatocytes. The damage score of the IR was significantly better in the levosimendan group. Şener et al. [38] administered melatonin in I/R and observed a protective effect on the liver parenchyma and hepatocytes, and no congestion was found in the sinusoidal structures. Abd-Elbaset [39] showed that thymoquinone alleviated damage due to I/R, such as inflammation in portal areas of the liver and focal spotty necrosis. Terzi et al. [40] found the protective effect of Urtica dioica by 

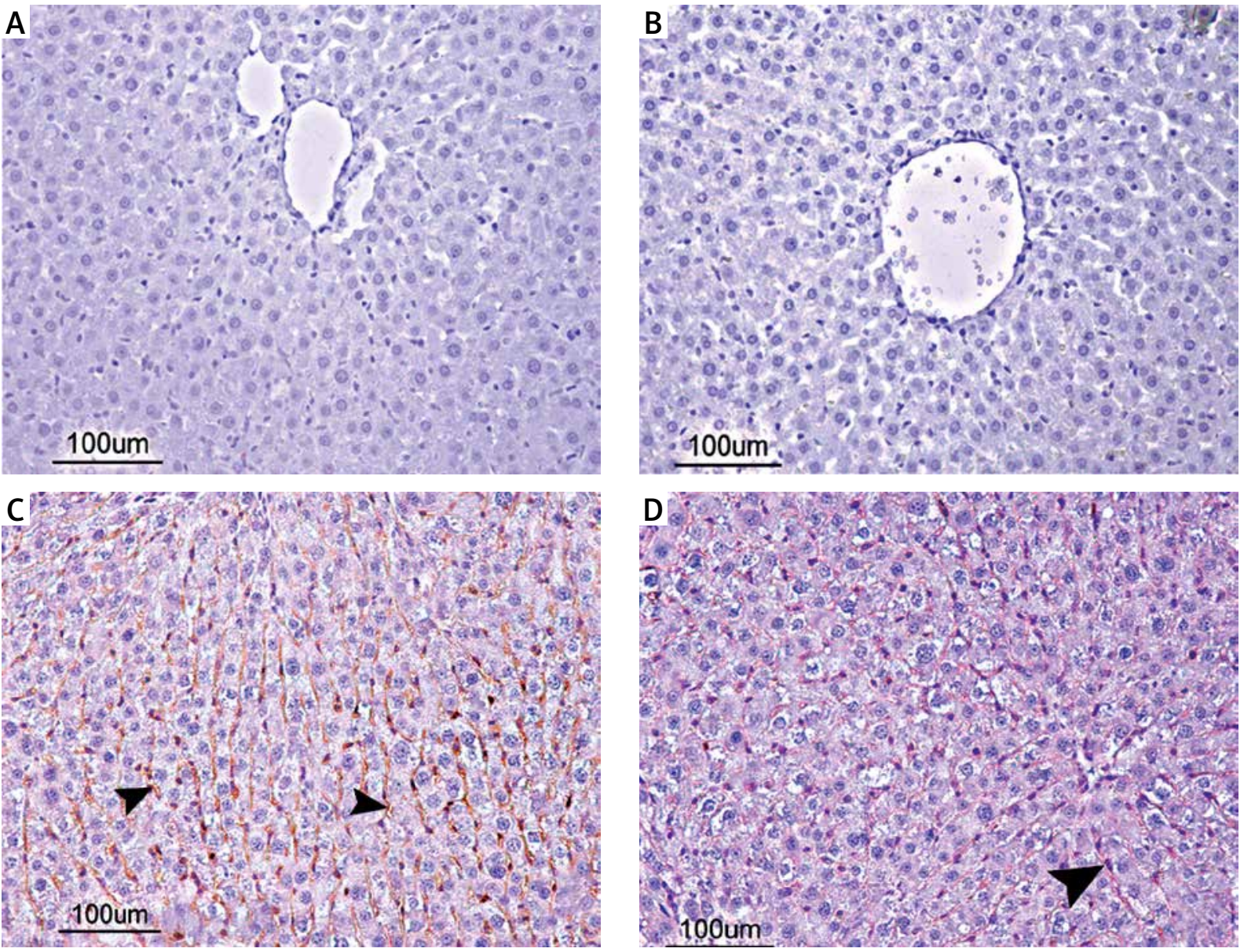

Figure 3. Photomicrographs of immunohistochemical investigation of the eNOS activity in liver. A, B - Negative eNOS activity of liver in the control group and AST group. C - Strong eNOS activity in the I/R group.

D - Low eNOS activity in the I/R + AST group

increasing the liver antioxidant capacity in an I/R study performed in rats. In this investigation on the liver histopathology of rats, in the I/R group parenchymal degeneration and hydropic degeneration, dilatation in the sinusoids and hyperaemia, coagulation necrosis with pycnotic nuclei in the hepatocytes, mononuclear cell infiltration in the periacinary and mid-zonal regions and in the portal area, structural deterioration in the hepatic cordons, and vascular congestion were revealed. In the $\mathrm{I} / \mathrm{R}+\mathrm{AST}$ group, congestion was minimal in the vena centralis and portal veins, and a low extent of dilatation in the sinusoids and insignificant sinusoidal hyperaemia were found. Generally, the hepatic cord structure in the lobules was not damaged, and it resembled that in the control group. No degeneration or necrosis in the hepatocytes and no mononuclear cell infiltration were observed. These findings are consistent with those of the above mentioned research in which different antioxidants were administered to avoid or lessen the damage caused by I/R.
To inactivate the free oxygen radicals forming in the kidney I/R models, agents such as iloprost [41], sodium hydrosulphide [42], red propolis [43], menhaden oil [17], and grape seed [44] were used. Additionally, Ozkan et al. [44] investigated the protective effect of grape seed proanthocyanidin in I/R damage. The study observed tubular vacuolisation, dilatation, congestion, and increased mononuclear cells in the kidney vascular structures, and administered proanthocyanidin to decrease them. Moreover, the decrease in I/R damage by this agent was due to its effect on lipid peroxidation and inflammatory cytokines, such as TNF- $\alpha$. Nigella sativa extract was also tested in the I/R setup by Yildiz et al. [45]. Its antioxidant property protected the kidneys by preventing dilatation of tubuli, brush border loss, and detachment of cells from the basal membrane. Maghsoudi et al. [46] tested Zingiber officinale extract in I/R and observed minimal dilatation in the tubules, degeneration in the tubular epithelial cells, and necrosis, compared with the groups without extract administration. 

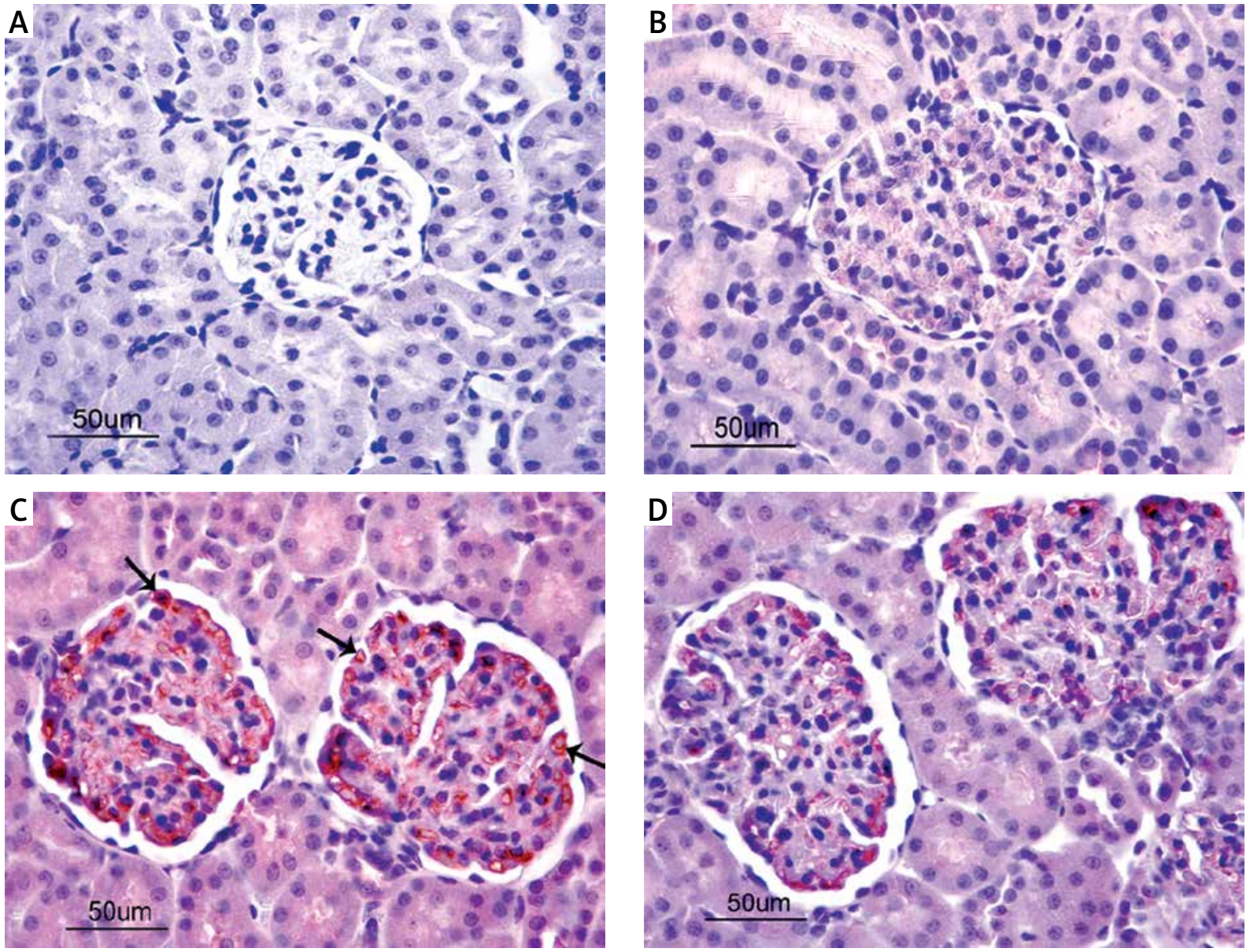

Figure 4. Photomicrographs of immunohistochemical investigation of the eNOS activity in kidney. A, B Negative eNOS activity of kidney in the control group and AST group. C - Strong eNOS activity in the I/R group. D - Low eNOS activity in the I/R + AST group

Table III. Protective effect of astaxanthin on liver and kidney tissue in experimental ischaemia reperfusion in rats as shown in eNOS activity

\begin{tabular}{lccc} 
Parameters (liver and kidney) & Control & I/R & I/R + AST \\
\hline Sinusoidal eNOS reactivity & - & +++ & ++ \\
\hline eNOS reactivity in glomerular and intertubular capillaries & - & +++ & +
\end{tabular}

In this study, the histopathological assessment of the I/R group revealed hydropic degeneration in the kidney tubuli epithelial cells, necrosis with pycnotic nuclei in some tubuli epithelial cells, dilatation in the tubules, dilatation and sclerosis in the glomeruli of Bowman capsules, adhesion in the glomeruli and Bowman capsules, and significant hyperaemia in the glomerular capillaries and intertubular region capillaries. Conversely, in the $I / R+A S T$ group, minimal hydropic degeneration in the tubular epithelial cells and minimal hyperaemia in the capillaries of some glomeruli and intertubular regions were found. Generally, the tubules and glomeruli seemed to be under normal conditions. This attenua-

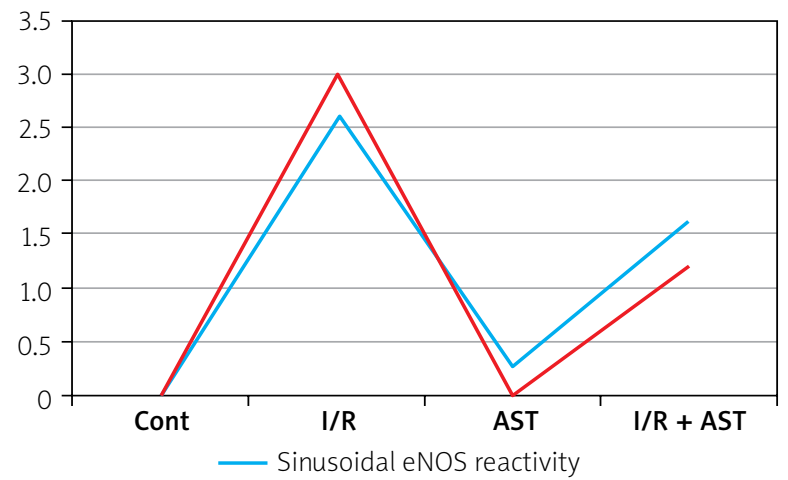

- eNOS reactivity in glomerular and intertubular capilaries

Figure 5. Mean values of groups for eNOS scores 
tion of kidney damage, which was also observed in liver tissue, was due to the potent antioxidant property of AST, which protected both tissues [47].

The decreased NO production in liver after I/R narrows the sinusoidal lumen, hampers the leucocyte movement, and causes leucocytosis by increasing the contact between leucocytes and endothelial cells. Following reperfusion, tissue remains ischaemic because of this condition occurring during hypoxia. Kupffer cells and neutrophils were activated by the produced inflammatory cytokines and the formation of free radicals due to the continued aggravation of hepatic damage of oxygen $[23,25]$. Several studies were conducted to show the eNOS activity following liver and kidney damage, because its decrease and imbalance with endothelin synthesis increase vascular resistance. Using tadalafil and pentoxifylline against liver I/R damage, Bektas et al. [48] observed the prevention of oxidative stress, lipid peroxidation, and hepatocyte damage and thus reported a minimisation of endothelial damage. Potent eNOS activity was observed in the I/R group. A moderate level of this activity was observed in the low-dose tadalafil group, whereas a weak eNOS activity was observed in the high-dose tadalafil and pentoxifylline group. Liver damage due to intestinal I/R was found to be prevented by sildenafil through its effect of avoiding oxidative stress [49]. In addition, a reversal of hepatic circulation deterioration was shown by eNOS immunohistochemically. Similarly, the contribution of eNOS was immunofluorescently shown in a study in which thymoquinone was used to prevent liver I/R damage [39].

In the pathophysiology of acute kidney diseases, such as acute kidney failure and I/R, endothelial and smooth muscle cells of the microcirculation play an important role $[26,50]$.

In a study that tested the effectiveness of red propolis in preventing kidney tubuli epithelium in I/R, eNOS levels were found to be significantly higher in the I/R group than in the control group, and red propolis ameliorated this increase significantly [43]. Similarly, quercetin [51] and sevoflurane [52] were found to be effective in avoiding oxidative stress in an I/R model.

The liver intrahepatic microvascular unit is composed of several discrete units, including portal and central veins, hepatic arterioles, sinusoids, and lymphatics. The cellular elements in these structures include endothelial cells and smooth muscle cells, and in the sinusoid, pericyte-like hepatic stellate cells [53]. Chronic exposure to physical and chemical stimuli can trigger endothelial dysfunction. Endothelial dysfunction can be defined as an impairment of normal endothelial functions, which is caused by the loss of balance between vasoconstrictors and vasodilators, growth promoting and inhibit- ing factors, pro-atherogenic and anti-atherogenic, and pro-coagulant and anti-coagulant factors. Endothelial dysfunction is also regarded as an early key event in multiple diseases [54], and it has also been considered as a pivotal event in the development of microvascular complications in chronic liver diseases, including liver cirrhosis, hypertension, diabetes, and atherosclerosis $[53,55]$. Chronic liver disease is associated with remarkable alterations in the intra- and extrahepatic vasculature [53]. In liver cirrhosis, on one hand, endothelial dysfunction, known to impair endothelium-dependent relaxation in the liver microcirculation, contributes to increased hepatic vascular resistance [56]. On the other hand, in arteries of the splanchnic circulation increased production of vasodilator molecules mainly endothelial nitric oxide synthase (eNOS)-driven nitric oxide (NO) plays a prime role in maintaining normal hepatic vascular function, which precedes the development of the hyperdynamic circulatory syndrome observed in cirrhosis with portal hypertension [57-59]. The present study aimed to show an increased eNOS enzyme activity caused by liver and kidney endothelial damage following I/R. In addition, it determined the sinusoidal capillaries in the liver and the glomerular and intertubular capillaries in the kidney through immunohistochemistry. Immunohistochemical activity was observed in the control group, and augmented staining, which is an indicator of increased immune reactivity, was observed in the I/R group. Minimal staining occurred in the group in which AST was administered prior to I/R. Attenuation of the liver and kidney damage occurred after reperfusion in the AST group, consistent with previous studies.

\section{Conclusions}

As a result, AST was shown to have protective effects in this experimental setup, in which tissue damage in liver and kidney due to I/R was investigated by histopathological and immunohistochemical methods. I/R-induced elevation in eNOS expression was reduced such that, in the case of acute lower extremity I/R, AST decreased the ischaemic injury in liver and renal tissues by protecting the microcirculation and providing a cytoprotective effect with vasodilatation. It was considered that this crucial protective role of AST might alleviate pathological changes.

\section{Acknowledgments}

We would like to thank to Prof. Dr. Zabit Yener for his expertise and assistance in microscopy.

All authors contributed equally to this work.

\section{Conflict of interest}

The authors declare no conflict of interest. 


\section{References}

1. Bonventre JV. Mechanism of ischemic akut renal failure. Kidney Int 1993; 43: 1160-78.

2. Carden DL, Granger DN. Pathophysiology of ischemia-reperfusion injury. J Pathol 2000; 190: 255-66.

3. Collard CD, Gelman S. Phatophysiology, clinical manifestations, and prevention of ischemia-reperfusion injury. Anesthesiology 2001; 94: 1133-8.

4. Majino G, Jorris I. Apopitosis, oncosis, and necrosis. An overview of the cell death. Am J Pathol 1995; 146: 3-9.

5. Zimmerman BJ, Granger N. Reperfusion injury. Surg Clin North Am 1992; 72: 65-83.

6. Ritenour AE, Christy RJ, Roe JL, et al. The effect of a hypobaric hypoxic environment on acute skeletal muscle edema after ischemia-reperfusion injury in rats. J Surg Res 2010; 15: 253-9.

7. Saba D, Yavuz H, Şenkaya I, et al. The effects of calcium dobesilate on skeletal muscle ischemia reperfusion injury. Turk J Thorac Cardiovasc Surg 2000; 8: 797-801.

8. Ferrari RS, Andrade CJ. Oxidative stress and lung ischemia-reperfusion injury. Oxid Med Cell Longev 2015; 2015 : 590987.

9. Jaeschke H, Smith CV, Mitchell JR. Reactive oxygen species during ischemia-reflow injury in isolated perfused rat liver I Clin Invest 1988; 81: 1240-6.

10. Weight SC, Bell PRF, Nicholson ML. Renal ischemia-reperfusion injury. Br J Surg 1996; 83: 162-70.

11. Majano PL, Medina J, Zubia I, et al. N-acetyl-cysteine modulates inducible nitric oxide synthase gene expression in human hepatocytes. J Hepatol 2004; 40: 632-7.

12. Zhang YH. Nitric oxide signalling and neuronal nitric oxide synthase in the heart under stress. F1000Res 2017; 6: 742.

13. Aydin I, Bulbul A, Avci GE, et al. Serum oxidative status and adenosine deaminase activity in dogs with transmissible venereal tumour. Bull Vet Inst Pulawy 2009; 53: 771-4.

14. Er A, Corum O, Cetin G, et al. Effect of Nerium oleander distillate administration on serum nitric oxide level. Eurasian J Vet Sci 2015; 31: 70-4.

15. Mutlu C, Koyutürk M, Karpuz V. Evaluation of endothelial nitric oxide synthase (eNOS) immunreactivity of fetal and materna placentaa. Cerrahpaşa J Med 2005; 36: 109-15.

16. Ergün O, Ulman C, Kılıçalp AS. Carnitine as a agent in experimental renal Ischemia-reperfusion Injury. J Urol Res 2001; 29: 186-9.

17. Ossani GP, Marcotegui AR, Uceda AM, et al. Menhaden oil rich diet and experimental renal damage due to ischemia reperfusion. J Oleo Sci 2017; 66: 1157-9.

18. Inglott FS, Virlos T, Habib NA, et al. Adenosine preconditioning attenuates hepatic reperfusion injury in the rat by preventing the down-regulation of endothelial nitric oxide synthase. BMC Gastroenterol 2002; 2: 22

19. Crockett ET, Galligan JJ, Bruce DU, et al. Protection of early phase hepatic ischemia-reperfusion injury by cholinergic agonists. BMC Clin Pathol 2006; 6: 3.

20. Peralta C, Bartrons R, Serafin A, et al. Adenosine monophosphate-activated protein kinase mediates the protective effects of ischemic preconditioning on hepatic ischemia-reperfusion injury in the rat. Hepatology 2001; 34: 1164-73.
21. Gedik E, Girgin S, Öztürk H, et al. Resveratrol attenuates oxidative stress and histological alterations induced by liver ischemia/ reperfusion in rats. World J Gastroenterol 2008; 14: 7101-6.

22. Gujral JS, Bucci TJ, Farhood A, et al. Mechanism of cell death during warm hepatic ischemia-reperfusion in rats: apoptosis or necrosis? Hepatology 2001; 33: 397-405.

23. Liu P, McGuire GM, Fisher MA, et al. Activation of Kupffer cells and neutrophils for reactive oxygen formation is responsible for endotoxin-enhanced liver injury after hepatic ischemia. Shock 1995; 3: 56-62.

24. Baykara B, Tekmen I. Karaciğer İskemi Reperfüzyon Hasarı. DEÜ Tıp Fakültesi Dergisi 2005; 19: 185-94.

25. Bonventre JV, Yang L Cellular pathophysiology of ischemic acute kidney injury. J Clin Invest 2011; 121: 4210-21.

26. Friedewald JJ, Rabb H. Inflammatory cells in ischemic acute renal failure. Kidney Int 2004; 66: 486-91.

27. Brady HR, Singer GG. Acute renal failure. Lancet 1995; 346 : 1533-40.

28. Conesa EL, Valero F, Nadal JC, et al. N-acetyl-L-cysteine improves renal medullary hypoperfusion in acute renal failure. Am J Physiol 2001; 281: R730-7.

29. Paller MS, Hoidal JR, Ferris TF. Oxygen free radicals in ischemic acute renal failure in rat. J Clin Invest 1984; 74: 1156-64.

30. Basile DP, Anderson MD, Sutton TA. Pathophysiology of acute kidney injury. Compr Physiol 2014; 2: 1303-53.

31. Carter SE, Foulkner A, Rakobowchuk M. The role of prostaglandin and antioxidant availability in recovery from forearm ischemia-reperfusion injury in humans. J Hypertens 2014; 32: 339-51.

32. Galaris D, Barbouti A, Korantzopoulos P. Oxidative stress in hepatic ischemia-reperfusion injury: the role of antioxidants and iron chelating compounds. Curr Pharm Des 2006; 12: 2875-90.

33. Ferramosca A, Di Giacomo M, Zara V. Antioxidant dietary approach in treatment of fatty liver: new insights and updates. World J Gastroenterol 2017; 21: 4146-57.

34. Chen JT, Kotani K. Astaxanthin as a potential protector of liver function: a review. J Clin Med Res 2016; 8: 701-4.

35. Vardanian AJ, Busuttil RW, Kepiec-Weglinski J. Molecular mediators of liver ischemia and reperfusion injury: a brief review. Mol Med 2008; 14: 337-45.

36. Lin CM, Lee JF, Chiang LL, et al. The protective effect of curcumin on ischemia-reperfusion-induced liver injury. Transplant Proc 2012; 44: 974-7.

37. Ahmetova A. Effects of levosimendan on experimental hepatic ischemia-reperfusion injury. Phd Thesis. Dokuz Eylül University, İzmir, 2010

38. Şener G, Tosun O, Sehirli AO, et al. Melatonin and N-acetylcysteine have benefical effects during hepatic ischemia and reperfusion. Life Sci 2003; 72: 2707-18.

39. Abd-Elbaset M, El-Shaimaa AA, El Sherbiny GA, et al. Thymoquinone mitigate ischemia-reperfusion-induced liver injury in rats: a pivotal role of nitric oxide signaling pathway. Naunyn-Schmiedeberg's Arch Pharmacol 2017; 390: 69-76.

40. Terzi A, Yıldız F, Çoban S, et al. Protective effect of urtica dioica on liver injury induced by hepatic ischemia reperfusion injury in rats. Duzce Med J 2010; 1: 43-7. 
41. Yakut N, Yasa H, Lafci BB, et al. The influence of levosimendan and iloprost on renal ischemia reperfusion: an experimental study. Interact Cardiovasc Thorac Surg 2008; 7: 235-9.

42. Ibrahim MY, Aziz NM, Kamel MY, et al. Sodium hydrosulphide against renal ischemia/reperfusion and the possible contribution of nitric oxide in adult male Albino rats. Bratisl Med J 2015; 116: 681-8.

43. da Costa MF, Libório AB, Teles F, et al. Red propolis ameliorates ischemic-reperfusion acute kidney injury. Phytomedicine 2015; 15: 787-95.

44. Ozkan G, Ulusoy S, Orem A, et al. Protective effect of the grape seed proanthocyanidin extract in a rat model of contrastinduced nephropathy. Kidney Blood Press Res 2012; 35: 445-53.

45. Yildiz F, Coban S, Terzi A, et al. Protective effects of Nigella sativa against ischemia-reperfusion injury of kidney. Ren Fail 2010; 32: 126-31.

46. Maghsoudi S, Gol A, Dabiri S, et al. Preventive effect of ginger (Zingiber officinale) pretreatment on renal ischemia-reperfusion in rats. Eur Surg Res 2011; 46: 45-51.

47. Özdamar MY, Yurtçu M, Toy H, et al. The effect of grape seed proanthocyanidin in renal ischemia-reperfusion injury. Genel Tip Derg 2010; 20: 1-5.

48. Bektas S, Karakaya K, Can M, et al. The effects of tadalafil and pentoxifylline on apoptosis and nitric oxide synthase in liver ischemia/reperfusion injury. Kaohsiung J Med Sci 2016; 32: 339-47.

49. Inan M, Uz YH, Kizilay G, et al. Protective effect of sildenafil on liver injury induced by intestinal ischemia/reperfusion. J Pediatr Surg 2013; 48: 1707-15.

50. Willams P, Lopez H, Britt D, et al. Characterization of renal ischemia-reperfusion injury in rats. J Pharmacol Toxicol Methods 1997; 37: 1-7.

51. Kinaci MK., Erkasap N, Kucuk A, et al. Effects of quercetin on apoptosis, NF-kappaB and NOS gene expression in renal ischemia/reperfusion injury. Exp Ther Med 2012; 3: 249-54.

52. Wang JM, Hu ZY, Gu WZ. Effects of sevoflurane postconditioning on renal ischemia-reperfusion injury: experiment with rats. Zhonghua Yi Xue Za Zhi 2009; 89: 1016-20.

53. Iwakiri Y, Shah V, Rockey DC. Vascular pathobiology in chronic liver disease and cirrhosis - current status and future directions. J Hepatol 2014; 61: 912-24.

54. Iwakiri Y, Grisham M, Shah V. Vascular biology and pathobiology of the liver: report of a single-topic symposium. Hepatology 2008; 47: 1754-63.

55. Svistounov D, Warren A, McNerney GP, et al. The relationship between fenestrations, sieve plates and rafts in liver sinusoidal endothelial cells. PLoS One 2012; 7: e46134.

56. Iwakiri Y, Groszmann RJ. Vascular endothelial dysfunction in cirrhosis. J Hepatol 2007; 46: 927-34.

57. Iwakiri Y. Endothelial dysfunction in the regulation of cirrhosis and portal hypertension. Liver Int 2012; 32: 199-213.

58. Wiest R, Shah V, Sessa WC, et al. NO overproduction by eNOS precedes hyperdynamic splanchnic circulation in portal hypertensive rats. Am J Physiol 1999; 276: G1043-51.

59. Vairappan B. Endothelial dysfunction in cirrhosis: role of inflammation and oxidative stress. World J Hepatol 2015; 7: 443-59.
Received: 24.04.2019

Accepted: 9.08.2019 